\title{
Virulence factors of uropathogenic Escherichia coli (UPEC) and correlation with antimicrobial resistance
}

\author{
Chhaya Shah", Ratna Baral ${ }^{1}$, Bijay Bartaula ${ }^{2}$ and Lok Bahadur Shrestha ${ }^{1 *}$
}

\begin{abstract}
Background: Escherichia coli has found to be the predominant uropathogen (50-90\%) in uncomplicated, community acquired urinary tract infection (UTI). Uropathogenic Escherichia coli (UPEC) express a multitude of virulence factors, which enable the bacteria to establish UTI. The objective of this study was to evaluate the presence of different phenotypic virulence markers in UPEC isolates and determine their correlation with antibiotic resistance pattern.

Results: Out of 105 patients, 56 (53\%) were females and 49 (47\%) were males. The age of the patients in the study ranged from 18 years to 87 years and majority of the patients belonged to the age group 20-29 years. Virulence factor was observed in 65\% ( $n=69)$ of UPEC and 20\% $(n=22)$ of control isolates ( $P=0.0001)$. Haemolysin production was observed in 34(32.3\%) of uroisolates and 12 (11.4\%) of control strain. Similarly, 62\% of UPEC and 1\% of control produced biofilm ( $P=0.0001)$. The expression of Mannose-resistant hemagglutinin $(M R H A)$ and mannosesensitive hemagglutinin (MSHA) in uroisolates were 52.3\% $(n=55)$ and $5.7 \%(n=6)$ respectively, whereas in faecal isolates, $8.5 \%(n=9)$ expressed MRHA and none produced MSHA. Antimicrobial resistance showed a high degree of resistance towards ampicillin, cotrimoxazole and norfloxacin. The resistance was observed in significant higher degree in biofilm formers as compared to non-formers. MDR and ESBL was observed in 51 and $46 \%$ of test strains and 9 and $7.6 \%$ of control strains $(P=0.0001)$.

Conclusion: A significant association between virulence factors of UPEC and antimicrobial resistance in UPEC was present. Routine testing of these factors and co-relation with AMR is recommended. These findings will certainly help understand the pathogenicity and proper management of UTI patients, thus decreasing the improper use of antibiotics.
\end{abstract}

Keywords: UPEC, MDR, Virulence factor, Hemagglutinin, Hemolysin

\section{Background}

Urinary tract infection (UTI) is the most commonly diagnosed urological and renal disease. It is frequently associated with morbidity in both hospitalized as well as outpatient [1]. In $50-90 \%$ of all the uncomplicated urinary tract infections, uropathogenic Escherichia coli (UPEC) is most common organism seen [2, 3]. UPEC are strains of Escherichia coli that divert from their commensal status as intestinal flora, grow and persist in the urinary tract and exhibit diverse array of virulence

\footnotetext{
* Correspondence: lok.shrestha@bpkihs.edu

'Department of Microbiology and Infectious Diseases, B. P. Koirala Institute of Health Sciences (BPKIHS), Sunsari, Dharan 56700, Nepal

Full list of author information is available at the end of the article
}

factors and strategies, which allow them to infect and cause diseases in the urinary tract. These strains of $E$. coli are consistently associated with uropathogenicity and are called as UPEC [4].

The important virulence factors of Escherichia coli can broadly be divided into two groups: bacterial cell surface and secreted virulence factor. Bacterial cell surface virulence factors most commonly include fimbriae like mainly type 1 fimbriae and $\mathrm{P}$ fimbriae. These fimbriae help in adhesion to host cell surface, tissue invasion (which is important in pathogenesis of UPEC causing UTIs), biofilm formation and cytokine induction. Bacterial cell surface virulence factor also include flagellum, capsular lipopolysaccharide and outer membrane proteins.

(c) The Author(s). 2019 Open Access This article is distributed under the terms of the Creative Commons Attribution 4.0 International License (http://creativecommons.org/licenses/by/4.0/), which permits unrestricted use, distribution, and 
Haemolysin and siderophores are secreted virulence factor. $[5,6]$. These virulence factors are important in enabling the bacteria to colonize the urinary tract and persist despite the effectively functioning host defense mechanism [7].

The emergence of drug resistant microorganism among UPEC strains increase the serious threat to global health [8]. Therefore, knowledge regarding local prevalence of UPEC and antimicrobial resistance is essential for optimal management of UTI. The study was thus designed with an objective to evaluate the presence of different phenotypic virulence markers in UPEC isolates and determine their correlation with antibiotic resistance pattern.

\section{Results}

During the study period, 1142 urine samples were obtained from internal medicine ward from same number of patients; 184 yielded bacterial growth with an infection rate of $16.11 \%$. Among them, 105 (57\%) were Escherichia coli. Out of 105 patients with UTI due to E. coli, 56 (53\%) were females and 49 (47\%) were males. The age of the patients in the study ranged from 18 years to 87 years. Majority of the patients belonged to the age group $20-29$ years $(36,34.28 \%)$ and $30-39$ years $(36,34.28 \%)$. One or more virulence factor was expressed by $65 \%(n=69)$ test strains and $6 \%(n=6)$ control strains. Hemolysin production was observed in $32.3 \%(n=34)$ of uroisolates and $11.4 \%(n=12))$ of control strain, which was statistically significant $(\mathrm{P}=0.002)$. Biofilm production was noted in $62 \%(n=65)$ of UPEC while only $1 \%(\mathrm{n}=1)$ control isolate produced biofilm $(\mathrm{P}<0.0001)$. Similarly, MRHA and MSHA were identified in $55(52.3 \%)$ and 6 (5.7\%) respectively in UPEC, while commensal E. coli showed 8.5\% $(\mathrm{n}=9)$ MRHA and no MSHA. Gelatinase production was detected in none of the isolates (Table 1).

Antimicrobial resistance was compared between the test strains and the commensal isolates. The test strains shows significantly higher degree of resistance as compared to the control strains (Table 2). Amikacin was resistant to $22 \%$ of UPEC, while only $10 \%$ resistance was noted in control strains $(\mathrm{P}=0.01)$. Norfloxacin resistance was noted in $46 \%$ of isolates and $15 \%$ of control strains $(\mathrm{P}=0.0001)$.

The correlation between the virulence factors along with the antimicrobial resistance was also analyzed (Table 3). The hemolysin producing strains showed a higher degree of resistance as compared to non-producing ones. Similar pattern of resistance was also observed among biofilm producing and non-producing strains. The level of resistance to amikacin (30\% vs $7.5 \%$, p value $=0.005)$, ampicillin $(67 \%$ vs $25 \%, P=0.0001)$, ceftriaxone $(49 \%$ vs $27 \%$, p value $=0.028)$, meropenem $(26 \%$ vs $10 \%, \mathrm{P}=0.044)$, nitrofurantoin $(38 \%$ vs $12.5 \%, \mathrm{P}=$ $0.04)$ and norfloxacin $(52 \%$ vs $30 \%, \mathrm{P}=0.041)$ was higher in biofilm formers than non-formers. Moreover, there was significant association found between the hemagglutinin positivity and resistance to antimicrobial agents.

Prevalence of MDR was found to be much higher in case of UPEC isolates as compared to the test strains (51\% vs $9 \%, \mathrm{P}=0.0001$ ). Similarly, comparison of MDR between virulence positive UPEC and virulence negative UPEC also showed significant differences (69\% vs $16 \%$, $\mathrm{P}=0.0001$ ). The comparative study of ESBL also showed similar result (Table 4).

\section{Discussion}

Characterization of UPEC isolates and their correlation with antibiotic resistance patterns in patients with UTI are not well known, particularly in Nepal. Understanding of virulence factors certainly aids in the proper management of UTI and eventually, the prevention of antimicrobial resistance.

In our study, UTI was more prevalent in females, with the total of 105 cases which constitutes 53\%. The result is in agreement with several other studies $[9,10]$. The incidence of UTI was more in the age group 20 to 39 with age group of the patient ranging from 17 years to 87 years. This result was supported by previous study of Hooton et al. [11], Kabugo et al. [12] and Jadhav et al. [13]. The possible explanation of this may be because most of these patients were female and women of the reproductive age group, who are most vulnerable, due to

Table 1 Virulence marker of UPEC and control

\begin{tabular}{|c|c|c|c|c|c|}
\hline & Virulence markers & UPEC (105) & Control (105) & $P$ value & Remarks \\
\hline 1 & Hemolysin production & $34(32.3 \%)$ & $12(11.4 \%)$ & $P=0.002$ & Significant \\
\hline 2. & Biofilm production & $65(62 \%)$ & $1(1 \%)$ & $P=0.0001$ & Significant \\
\hline \multirow[t]{3}{*}{3.} & Hemagglutinin & & & $P<0.0001$ & Significant \\
\hline & MRHA & $55(52.3 \%)$ & $9(8.5 \%)$ & & \\
\hline & MSHA & $6(5.7 \%)$ & $0(0 \%)$ & & \\
\hline 4. & Gelatinase & 0 & 0 & NA & \\
\hline
\end{tabular}


Table 2 Comparison of antibiotic resistance (\%) between UPEC and commensal isolates

\begin{tabular}{llll}
\hline Antibiotic & UPEC $(n=105)$ & Commensals $(n=105)$ & $P$ value \\
\hline Amikacin & 22 & 10 & 0.01 \\
Ampicillin & 51 & 30 & 0.002 \\
Ceftriaxone & 40 & 20 & 0.0016 \\
Cotrimoxazole & 46 & 15 & 0.0001 \\
Meropenem & 20 & 2 & 0.0001 \\
Nitrofurantoin & 28 & 12 & 0.0038 \\
Norfloxacin & 43 & 15 & 0.0001 \\
\hline
\end{tabular}

short urethra, or close proximity of the urethra to anal canal and vagina $[4,14]$.

Our study showed greater production of hemolysin by UPEC $(32.3 \%, \mathrm{n}=34)$ than the control strains $(11.4 \%$, $\mathrm{n}=12)$, which is statistically significant $(\mathrm{P}<0.0001)$. This finding is consistent with several studies done previously $[13,15,16]$. A similar study done by Mittal et al. [9] revealed a higher rate of hemolysin production(47\%) in UPEC, while Kauser et al. [17] observed only $21 \%$ were hemolysin producer. The toxin, hemolysin, is a vital virulence factor of UPEC which targets multiple host pathways to facilitate infection [18].

In the present study, biofilm formation was evident in majority of UPEC; this is in agreement with study done by Karam et al. [15], Sharma et al. [19] and Dumaru et al. [20]. Study done by Fattahi et al. [21] have observed a higher rate of biofilm formation (90\%). Soto et al. [22] and Karam et al. [15] concluded that biofilm formation was higher in UPEC than in control strains, which is in concordance with our finding. Biofilm is one of the important virulence factors of UPEC protect them from host immunity and antimicrobial agents causing persistent infections [22].

We observed a notable difference in MRHA expression in test isolates as compared to the control (52.3\% vs $8.5 \%, \mathrm{P}=0.0001$ ); the results are consistent with study done by Raksha et al. [2], Najar et al. [23] and Vagarali et al. [7]. The type 1 fimbriae, MSHA was expressed in $5.7 \%$ of UPEC and none of the commensal strains, as per our research. Kaira et al. [24] demonstrated expression of $41 \%$ MRHA and 5\% MSHA in their study; the results are in agreement with our finding. Fimbriae (P fimbriae and type 1 fimbriae) plays an important role in pathogenesis of UTI by facilitating the attachment of $E$. coli to the uroepithelium [7].

None of the isolates in our study produced gelatin, which was in accordance to study by Kaira et al. where none of the isolates showed gelatinase production and EL-Mosallamy et al. where only $1(2 \%)$ showed gelatinase production [24, 25]. However, another study has demonstrated a high degree of gelatin production [9]. Gelatinases is an important virulence factor of $E$. coli responsible for pathogenicity in different diseases, particularly UTI $[2,5]$.

The study of antimicrobial resistance showed a variable degree of resistance among UPEC isolates; 51\% were resistant to ampicillin, $43 \%$ to norfloxacin and $20 \%$ to Meropenem. The comparison of antimicrobial resistance among UPEC and control strains showed a significant difference. The results agree with the study done by Karam et al. [15]. and $\mathrm{Li}$ et al. [26]. Multi-drug

Table 3 Relationship between antimicrobial resistance and virulence factor genes in UPEC

\begin{tabular}{|c|c|c|c|c|c|c|c|}
\hline \multirow[b]{2}{*}{ Virulence marker } & \multicolumn{7}{|c|}{ Antibiotic resistance (\%) } \\
\hline & Amikacin & Ampicillin & Ceftriaxone & Cotrimoxazole & Meropenem & Nitrofurantoin & Norfloxacin \\
\hline \multicolumn{8}{|l|}{ Hemolysin } \\
\hline Positive $(n=34)$ & 41 & 88 & 73 & 85 & 35 & 50 & 70 \\
\hline Negative $(n=71)$ & 12 & 33 & 25 & 28 & 12 & 18 & 30 \\
\hline$P$ value & 0.001 & 0.0001 & 0.0001 & 0.0001 & 0.007 & 0.001 & 0.003 \\
\hline \multicolumn{8}{|l|}{ Biofilm } \\
\hline Positive $(n=65)$ & 30 & 67 & 49 & 60 & 26 & 38 & 52 \\
\hline Negative $(n=40)$ & 7.5 & 25 & 27 & 25 & 10 & 12.5 & 30 \\
\hline$P$ value & 0.005 & 0.0001 & 0.028 & 0.375 & 0.044 & 0.04 & 0.041 \\
\hline \multicolumn{8}{|l|}{ Hemagglutinin } \\
\hline MRHA positive $(n=55)$ & 32 & 69 & 52 & 67 & 23 & 36 & 58 \\
\hline MRHA negative $(n=50)$ & 10 & 32 & 28 & 24 & 16 & 20 & 28 \\
\hline$P$ value & 0.005 & 0.001 & 0.01 & 0.126 & 0.329 & 0.064 & 0.012 \\
\hline MSHA positive $(n=6)$ & 66 & 83 & 83 & 83 & 50 & 83 & 83 \\
\hline MSHA negative $(n=99)$ & 19 & 49 & 38 & 44 & 18 & 25 & 41 \\
\hline$P$ value & 0.006 & 0.088 & 0.030 & 0.001 & 0.05 & 0.002 & 0.179 \\
\hline
\end{tabular}


Table 4 Comparison of MDR and ESBL between test and control, virulence positive and negative UPEC

\begin{tabular}{lllllll}
\hline & UPEC $(n=105)$ & Commensal $(n=105)$ & P value & Virulence Positive UPEC $(n=69)$ & Virulence negative UPEC $(n=36)$ & $P$ value \\
\hline MDR & $51 \%(n=54)$ & $9 \%(n=10)$ & 0.0001 & $69 \%(n=48)$ & $16 \%(n=6)$ & 0.0001 \\
ESBL & $46 \%(n=49)$ & $7.6 \%(n=8)$ & 0.0001 & $63 \%(n=44)$ & $13 \%(n=5)$ & 0.0001
\end{tabular}

resistance was observed at a higher rate among UPEC strains as compared to control (51\% vs $9 \%, \mathrm{P}=0.0001)$. Similarly, ESBL production was significantly higher in test strains in comparison with the commensals (46\% vs 7.6\%). The findings are similar to study conducted by Tabasi et al. [8] and Karam et al. [15]. Other studies have shown similar prevalence of ESBL production among UPEC [27, 28]. Jadhav et al. (21.3\%) have reported a lower incidence of ESBL and Mittal et al. (29.62\%) have reported lower incidence of $\operatorname{MDR}[9,13]$. The higher rate of MDR and ESBL in our setting might be due to the easy availability of antimicrobial as over the counter medication in our country.

Antimicrobial resistance was significantly higher in biofilm producing UPEC as compared to biofilm non-producers. The finding is in concordance with studies done by Karam et al. [15], Tadepalli et al. [29] and Tabasi et al. [8]. Several studies done in the past also conclude the same findings [30, 31]. Pompilio et al. [32] concluded that, although antimicrobial resistance was higher in biofilm producers, the difference was statistically non-significant. Protection of microorganism from host immunity, insufficient antimicrobial concentration and delayed penetration into the deeper layers of biofilms are the major reasons for antibiotic resistance in biofilm structures [22].

The study emulated a significant correlation between the hemolysin production and antimicrobial resistance. Amikacin was resistant in $41 \%$ of hemolysin positive strains while only $12 \%$ resistance was observed in hemolysin negative strains $(\mathrm{P}=0.0001)$. Similar pattern of resistance was observed against other antimicrobial agents. Similarly, the UPEC isolates demonstrating $\mathrm{P}$ fimbriae (MRHA) and Type 1 fimbriae (MSHA) showed higher degree of resistance than the ones without these virulence factors. The findings are similar to studies conducted in the past $[8,15]$. Fimbriae (Type 1 fimbriae and $P$ fimbriae) help in adhesion, quorum sensing and biofilm development which directly or indirectly contributes to the development of resistance to antimicrobials [33].

The prevalence of MDR (69\% vs $16 \%, \mathrm{P}=0.0001)$ and ESBL $(63 \%$ vs $13 \%, \mathrm{P}=0.0001)$ was markedly higher in virulent UPEC as compared to non-virulent UPEC strains. The findings are similar to study done by Jadhav et al. [13], Karam et al. [15] and Tabasi et al. [8].

\section{Conclusion}

We found a very significant association between virulence factors of UPEC and antimicrobial resistance in
UPEC. Routine testing of these factors and co-relation with AMR is recommended. These findings will certainly help understand the pathogenicity and proper management of UTI patients, thus decreasing the improper use of antibiotics.

\section{Methods}

This is a prospective study, which was conducted in a period of one year from September 2017 to August 2018 in the department of Microbiology and infectious diseases in collaboration with department of Internal medicine at B.P. Koirala Institute of health sciences (BPKIHS) Dharan, Nepal. Urine samples were obtained from patients from Internal medicine unit who were symptomatic for UTI. A total of 105 isolates of Escherichia coli were taken from the urine and the same number of control were used (faecal isolates from apparently healthy individuals). The isolates were identified by standard microbiological methods.

\section{Detection of hemolysin production $[2,8,34]$}

The organism from the bacterial stock vial was inoculated into peptone water till turbidity matching $0.5 \mathrm{Mc}$ Farland was obtained. Thereafter the organism was inoculated onto $5 \%$ sheep blood agar and incubated overnight at $35^{\circ} \mathrm{C}$. Hemolysin production was detected by complete hemolysis or Beta hemolysis of the erythrocytes on blood agar indicated by clearing around the colony. Plate was also kept at 4 degree centigrade overnight to clearly observe the hemolysis.

\section{Biofilm production [35]}

Biofilm production was detected by using congo red gar method proposed by Freeman et al. [35]. Congo red agar was prepared by mixing brain heart infusion broth, sucrose, congo red dye and agar (HiMedia) in 11 distilled water. The organisms were plated on it and incubated aerobically at $37^{\circ} \mathrm{C}$ for $24 \mathrm{~h}$. The observation of black colored colony was considered as biofilm positive and red colored colony as negative.

\section{Hemagglutination test $[9,34,36]$}

The test was performed according to the direct bacterial hemagglutination test-slide method. Human blood group 'O' obtained from the blood transfusion service was centrifuged and washed thrice in $0.85 \%(\mathrm{w} / \mathrm{v}) \mathrm{NaCl}$ solution (saline) and made into 3\% RBC suspension. Colonies from overnight growth of $E$. coli from the plate, were 
inoculated into $1 \%$ nutrient broth. It was then incubated for $48 \mathrm{~h}$ till full fimbriation. It was centrifuged and the bacillary deposits obtained were re-suspended in a small amount of residual fluid.

One drop of 3\% RBC suspension was added to a drop of the bacillary culture. The tile was rocked at room temperature by tilting it to and for $10 \mathrm{~min}$. If clumping was positive, it was said to be positive for hemagglutination. In a parallel test, a drop of $2 \%(\mathrm{w} / \mathrm{v})$ D-mannose was added to bacillary culture and 3\% RBC for which absence of hemagglutination was taken as MSHA) and presence of hemagglutination as MRHA.

\section{Gelatinase test $[9,34]$}

The test was done by gelatin plate method where gelatin nutrient agar was used to test for gelatinase production by the bacteria. The plate was inoculated with the heavy inoculum of an overnight old strain of Escherichia coli. The growth of the organism was observed and thereafter plate was flooded with mercuric chloride solution. The development of opacity in the medium and a zone of clearing around the colonies on flooding with mercuric chloride solution is indicative of gelatin liquefying colony and was considered positive for gelatinase.

\section{Antimicrobial susceptibility testing [37]}

It was performed according to standards of Clinical and laboratory Standards Institute (CLSI) guideline using Kirby Bauer's disc diffusion method on Muller-Hilton agar (HiMedia laboratories). Antibiotics discs such as amikacin $30 \mu \mathrm{g}$, ampicillin $10 \mu \mathrm{g}$, ceftriaxone $30 \mu \mathrm{g}$, Norfloxacin $10 \mu \mathrm{g}$, cotrimoxazole (trimethoprim/ sulfamethoxazole) $1.25 \mu \mathrm{g} / 23.75 \mu \mathrm{g}$, Meropenem $10 \mu \mathrm{g}$, nitrofurantoin $300 \mu \mathrm{g}$ were tested.

\section{Statistical analysis}

Data were collected and entered into a database using MS Excel 2013. Statistical analysis was conducted using SPSS version 16. Chi-square test was applied for comparison of categorical variables. The strength of the relationship and their 95\% of confidence interval was calculated. $\mathrm{P}$ value less than 0.05 was considered as statistically significant.

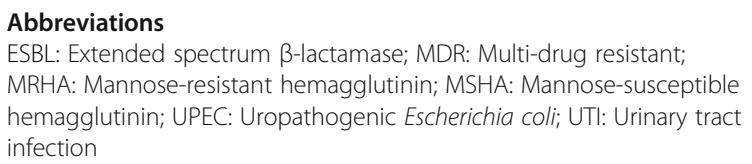

\section{Acknowledgements}

We would like to acknowledge Prof. Dr. Basudha Khanal, Head, Microbiology for her continuous support during the study.
Authors' contributions

Conceptualization: CS, RB, LBS. Investigation: CS. Resources: RB, BB, LBS.

Supervision: RB, BB, LBS. Writing-original draft: CS. Writing-review and editing:

LBS. All authors read and approved the final manuscript.

Funding

None.

Availability of data and materials

Yes, available from corresponding author upon request.

Ethics approval and consent to participate

Was obtained from Institutional Review Committee (IRC), B. P. Koirala Institute of Health Sciences, Dharan, Sunsari, Nepal.

Code no IRC/1120/017.

Consent to participate: written informed consent was obtained from each patient.

Consent for publication

Not applicable.

\section{Competing interests}

We declare that we have no competing interests.

\section{Author details}

${ }^{1}$ Department of Microbiology and Infectious Diseases, B. P. Koirala Institute of Health Sciences (BPKIHS), Sunsari, Dharan 56700, Nepal. ${ }^{2}$ Department of Internal Medicine, B. P. Koirala Institute of Health Sciences (BPKIHS), Sunsari, Dharan 56700, Nepal.

Received: 19 February 2019 Accepted: 28 August 2019

Published online: 02 September 2019

References

1. Nicolle LE. Epidemiology of urinary tract infections. Clin Microbiol Newsl. 2002;24(18):135-40.

2. Raksha R, Srinivasa $H$, Macaden RS. Occurrence and characterisation of uropathogenic Escherichia coli in urinary tract infections. Indian J Med Microbiol. 2003;21(2):102-7.

3. Foxman B. Urinary tract infection syndromes: occurrence, recurrence, bacteriology, risk factors, and disease burden. Infect Dis Clin N Am. 2014; 28(1):1-13.

4. Foxman B, Barlow R, D'Arcy H, Gillespie B, Sobel JD. Urinary tract infection: self-reported incidence and associated costs. Ann Epidemiol. 2000;10(8): 509-15.

5. Johnson JR. Virulence factors in Escherichia coli urinary tract infection. Clin Microbiol Rev. 1991;4(1):80-128. [358180]

6. Emody L, Kerenyi M, Nagy G. Virulence factors of uropathogenic Escherichia coli. Int J Antimicrob Agents. 2003;22(Suppl 2):29-33.

7. Vagarali MA, Karadesai SG, Patil CS, Metgud SC, Mutnal MB. Haemagglutination and siderophore production as the urovirulence markers of uropathogenic Escherichia coli. Indian J Med Microbiol. 2008;26(1):68-70.

8. Tabasi M, Asadi Karam MR, Habibi M, Yekaninejad MS, Bouzari S. Phenotypic assays to determine virulence factors of Uropathogenic Escherichia coli (UPEC) isolates and their correlation with antibiotic resistance pattern. Osong public health and research perspectives. 2015;6(4):261-8. [4588432]

9. Mittal S, Sharma M, Chaudhary U. Study of virulence factors of uropathogenic Escherichia coli and its antibiotic susceptibility pattern. Indian J Pathol Microbiol. 2014;57(1):61-4.

10. Foxman B. Epidemiology of urinary tract infections: incidence, morbidity, and economic costs. Am J Med. 2002;113(Suppl 1A):5S-13S.

11. Hooton TM, Scholes D, Hughes JP, Winter C, Roberts PL, Stapleton AE, et al. A prospective study of risk factors for symptomatic urinary tract infection in young women. N Engl J Med. 1996;335(7):468-74.

12. Kabugo D, Kizito S, Ashok DD, Graham KA, Nabimba R, Namunana $S$, et al. Factors associated with community-acquired urinary tract infections among adults attending assessment Centre. Mulago Hospital Uganda Afr Health Sci. 2016;16(4):1131-42. [5398460]

13. Jadhav S, Hussain A, Devi S, Kumar A, Parveen S, Gandham N, et al, Virulence characteristics and genetic affinities of multiple drug resistant 
uropathogenic Escherichia coli from a semi urban locality in India. PLoS One. 2011;6(3):e18063. [3064663]

14. Hooton TM. Recurrent urinary tract infection in women. Int J Antimicrob Agents. 2001;17(4):259-68.

15. Karam MRA, Habibi M, Bouzari S. Relationships between virulence factors and antimicrobial resistance among Escherichia coli isolated from urinary tract infections and commensal isolates in Tehran, Iran. Osong public health and research perspectives. 2018;9(5):217-24. [6202021]

16. Navidinia M, Peerayeh SN, Fallah F, Bakhshi B, Sajadinia RS. Phylogenetic grouping and pathotypic comparison of urine and fecal Escherichia coli isolates from children with urinary tract infection. Braz J Microbiol. 2014 45(2):509-14. [4166276]

17. Kauser Y, Chunchanur SK, Nadagir SD, Halesh LH, Chandrashekhar MR. Virulence factors, serotypes \& antimicrobial susceptibility of Escherichia coli in urinary tract infection. Al amen journal of medical sciences. 2009; 2:47-1. Al Amen Journal of Medical Sciences. 2009;2:47-1.

18. Justice SS, Hunstad DA. UPEC hemolysin: more than just for making holes. Cell Host Microbe. 2012;11(1):4-5. [3267088]

19. Sharma M, Aparna, Yadav S, Chaudhary U. Biofilm production in uropathogenic Escherichia coli. Indian J Pathol Microbiol. 2009;52(2):294.

20. Dumaru R, Baral R, Shrestha LB. Study of biofilm formation and antibiotic resistance pattern of gram-negative bacilli among the clinical isolates at BPKIHS. Dharan BMC Res Notes. 2019;12(1):38. [6339267]

21. Fattahi S, Kafil HS, Nahai MR, Asgharzadeh M, Nori R, Aghazadeh M. Relationship of biofilm formation and different virulence genes in uropathogenic Escherichia coli isolates from Northwest Iran. GMS hygiene and infection control. 2015;10:Doc11. [4512245].

22. Soto SM, Smithson A, Horcajada JP, Martinez JA, Mensa JP, Vila J. Implication of biofilm formation in the persistence of urinary tract infection caused by uropathogenic Escherichia coli. Clin Microbiol Infect. 2006;12(10): 1034-6.

23. Najar G, Najed M, Mansouri S. Nejad MM, Mansouri S. The comparison between virulence factors of Escherichia coli isolated from urinary tract infections and feacal flora. Res Pharma Sci 2007:2:99-103.

24. Kaira S, Pai C. Study of uropathogenic Escherichia coli with special reference to its virulence factors. Int J Community Med Public Health. 2018;1(1):177-81.

25. EL-Mosallamy WA, Desouky SM, El-Azum AAA, El Hamid HSA. Detection of some virulence factors and pyelonephritis-associated pilus (pap) encoding operon gene in Uropathogenic Escherichia coli. Egypt J Med Microbiol 2015;24(3):37-43

26. Li B, Zhao ZC, Wang MH, Huang XH, Pan YH, Cao YP. Antimicrobial resistance and integrons of commensal Escherichia coli strains from healthy humans in China. J Chemother. 2014;26(3):190-2. [4014832]

27. Shrestha LB, Baral R, Poudyal P, Khanal B. Clinical, etiological and antimicrobial susceptibility profile of pediatric urinary tract infections in a tertiary care hospital of Nepal. BMC Pediatr. 2019;19(36).

28. Parajuli NP, Maharjan P, Parajuli H, Joshi G, Paudel D, Sayami S, et al. High rates of multidrug resistance among uropathogenic Escherichia coli in children and analyses of ESBL producers from Nepal. Antimicrob Resist Infect Controll. 2017;6:9. [5225645]

29. Tadepalli S, Prudhivi S, Babu Myneni R, Rao S. Biofilm formation in uropathogenic Escherichia coli isolates and its association with extended spectrum betalactamase production and drug resistance. Saudi J Pathol Microbiol. 2016;1 (2):60-4

30. Shrestha LB, Bhattarai NR, Khanal B. Antibiotic resistance and biofilm formation among coagulase-negative staphylococci isolated from clinical samples at a tertiary care hospital of eastern Nepal. Antimicrob Resist Infect Controll. 2017;6:89. [5579930]

31. Shrestha LB, Bhattarai NR, Khanal B. Comparative evaluation of methods for the detection of biofilm formation in coagulase-negative staphylococci and correlation with antibiogram. Infect Drug Resist. 2018;11:607-13. [5926075]

32. Pompilio A, Crocetta V, Savini V, Petrelli D, Di Nicola M, Bucco S, et al. Phylogenetic relationships, biofilm formation, motility, antibiotic resistance and extended virulence genotypes among Escherichia coli strains from women with community-onset primitive acute pyelonephritis. PLoS One. 2018;13(5):e0196260. [5951556]

33. Thakur P, Chawla R, Tanwar A, Chakotiya AS, Narula A, Goel R, et al. Attenuation of adhesion, quorum sensing and biofilm mediated virulence of carbapenem resistant Escherichia coli by selected natural plant products. Microb Pathog. 2016;92:76-85.
34. Shruthi N, Kumar R, Kumar R. Phenotypic study of virulence factors in Escherichia coli isolated from antenatal cases, catheterized patients, and faecal flora. J Clin Diagn Res. 2012;6(10):1699-703. [3552208]

35. Freeman DJ, Falkiner FR, Keane CT. New method for detecting slime production by coagulase negative staphylococci. J Clin Pathol. 1989;42(8): 872-4. [1142068]

36. Ljungh A, Faris A, Wadstrom T. Hemagglutination by Escherichia coli in septicemia and urinary tract infections. J Clin Microbiol. 1979;10(4):477-81. [273199]

37. CLSI. Performance standards for antimicrobial susceptibility testing $-26^{\text {th }}$ edition. CLSI document M100S. Clinical and Laboratory Standards Institute: Wayne, PA; 2016.

\section{Publisher's Note}

Springer Nature remains neutral with regard to jurisdictional claims in published maps and institutional affiliations.
Ready to submit your research? Choose BMC and benefit from:

- fast, convenient online submission

- thorough peer review by experienced researchers in your field

- rapid publication on acceptance

- support for research data, including large and complex data types

- gold Open Access which fosters wider collaboration and increased citations

- maximum visibility for your research: over $100 \mathrm{M}$ website views per year

At BMC, research is always in progress.

Learn more biomedcentral.com/submissions 